\title{
Expansión urbana, humedales y evolución en los usos del suelo en el Gran La Plata
}

\author{
ISABel LóPez', Daniela RotgeR ${ }^{1,2}$ \\ ${ }^{1}$ Centro de Investigaciones Urbanas y Territoriales (CIUT-FAU-UNLP), La Plata, Argentina. \\ ${ }^{2}$ Consejo Nacional de Investigaciones Científicas y Tecnológicas, (CONICET). \\ E-mail: ilopez.arqui@gmail.com
}

\begin{abstract}
RESUMEN. Junto con la fundación de la ciudad de La Plata, en tierras aledañas de muy buena calidad se previeron actividades agropecuarias, que no sólo abastecen hasta la actualidad a las ferias barriales, sino que este cinturón productivo provee de productos frescos al Gran La Plata y la Región Metropolitana de Buenos Aires. La tecnología empleada para la producción de alimentos ha cambiado sustancialmente, así como la utilización del suelo, en la que compiten en extremo la presión urbanizadora y las actividades hortícolas y florísticas. Los instrumentos de planificación en la ciudad no acompañaron el desarrollo de la producción intensiva, y lo urbano fue extendiéndose sobre lo rural, ocasionando la pérdida de diversidad en los cultivos y el deterioro de valiosos ecosistemas, como los bañados de desborde fluvial. El objetivo de este artículo es realizar una revisión de los conflictos ambientales que han llevado al deterioro de los ecosistemas y el suelo en la ciudad, considerando como unidades ambientales-territoriales las sub-cuencas de arroyos, abordando las estrategias que podrían emplearse para mejorar la calidad de vida en las áreas periurbanas y rurales. La contigüidad en forma de ciudad difusa desde el urbanismo, sobre los bañados o cañadas que dan origen a los arroyos, así como el uso de agroquímicos y la falta de servicios básicos, aumenta la degradación ambiental, el riesgo de inundación por falta de superficie de infiltración, anula los servicios ecosistémicos contribuye a la reducción o desaparición de los humedales. Es necesario promover la coexistencia de ambos ámbitos, siendo que lo rural puede favorecerse de la accesibilidad que otorga lo urbano, que incorpora vías de comunicación y transporte público.
\end{abstract}

Palabras clave: Bañados, expansión urbana, Gran La Plata, matriz periurbana y rural, usos del suelo.

ABSTRACT. Together with the foundation of the city of La Plata, agricultural activities were planned in the surrounding lands that were of very good quality. This productive belt does not supply neighbourhood fairs to date but also provides fresh products to Great La Plata and the Metropolitan Region of Buenos Aires. The technology used for food production has changed, as well as the use of the land, in which urbanized pressure and horticultural and floristic activities are extremely competitive. The planning instruments in the city did not accompany the development of intensive production, and the urban environment spread over the rural, causing the loss of diversity in crops and the deterioration of valuable ecosystems, such as the wetlands. The main objective of this article is to unravel the environmental conflicts that resulted in the damage of ecosystems and soil in the city, considering the sub-basins of streams as environmental-territorial units, addressing the strategies that could be used to improve the quality of life in peri-urban and rural areas. The contiguity in the form of a diffuse city from urban planning on wetlands that give rise to streams, as well as the use of agrochemicals and the lack of basic services, increases environmental degradation and the risk of flooding due to lack of infiltration surface, nullifies ecosystem services and causes wetlands to reduce or disappear. It is necessary to promote the coexistence of both areas, since the rural can benefit from the accessibility that the urban provides through communication routes and public transport.

Keywords: Greater La Plata, land uses, peri-urban and rural matrix, urban expansion, wetlands. 


\section{Proceso de expansión urbana del Gran La Plata}

El Gran La Plata (GLP) (Figura 1) es un conglomerado urbano situado sobre el litoral sur de la Región Metropolitana de Buenos Aires (RMBA), formado por los partidos de La Plata, Berisso, Ensenada y Puerto La Plata. La región cuenta con una población de 801901 habitantes en base a proyecciones del Censo Nacional del año 2010 (INDEC, 2010). De esa población, 654112 habitantes pertenecen al partido de La Plata, 89096 al de Berisso y 58693 habitantes al partido de Ensenada (López et al., 2016). La ciudad de La Plata, como cabecera del GLP, fue fundada sobre el paraje denominado en agrícola, ganadera y portuaria, el paisaje original descrito ya se encontraba transformado, sin embargo la fundación de la ciudad de La Plata en 1882 como capital de la Provincia de Buenos Aires dio lugar a profundas modificaciones del relieve natural.

El proceso de organización urbana de La Plata puede resumirse en cuatro grandes etapas relacionadas con los procesos de expansión de la RMBA. Una primera etapa que abarca hasta la década del '30 del siglo $X X$, una segunda que comprende el proceso de industrialización iniciado por la sustitución de importaciones (1930 - 1955), la tercera delimitada por la profundización de la sustitución de importaciones (1955 - 1976), y
PAÍS: ARGENTINA PROVINCIA: BUENOS AIRES REGIÓN METROPOLITANA DE BUENOS AIRES
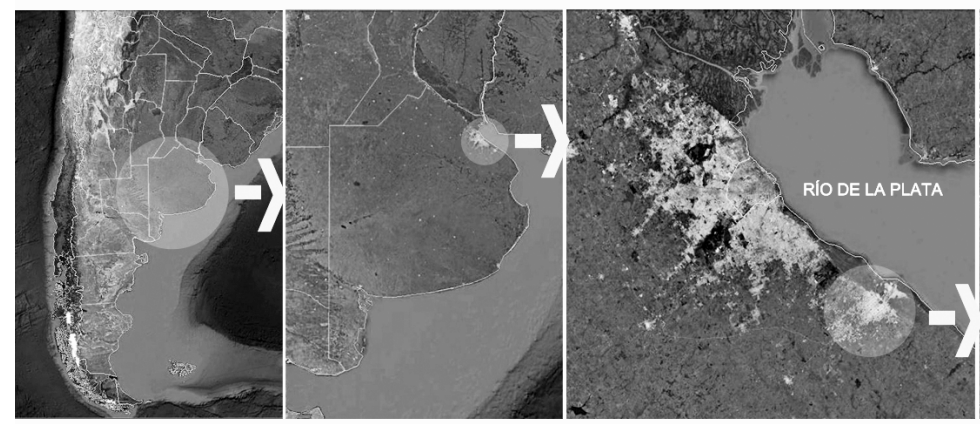

GRAN LA PLATA

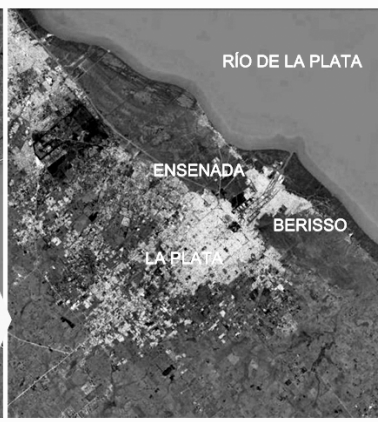

Figura 1. Localización regional del Gran La Plata. Fuente: Rotger et al., 2018.

Figure 1. Regional location of Greater La Plata.

1882 como "Lomas de Ensenada", localizada en la pampa ondulada, con una altitud variable entre los 5 y los 22,5 ms.n.m. dentro del casco fundacional. El paisaje primitivo de la región estaba caracterizado por el estuario del Río de la Plata, la pampa ondulada, la franja de bañados y el albardón costero, como límite de la costa del estuario. Las tres comunidades vegetales que existían en la región eran el manto cespitoso, el talar y en algunos sectores la selva marginal rioplatense. Además, existía un conjunto de arroyos que llevaban al estuario del río las aguas pluviales, entre los que las crónicas fundacionales destacan se hallan El Pescado, cercano a Magdalena, y del Gato, ubicado en la zona elegida para fundar la ciudad de La Plata (Rotger y López, 2019).

Ya desde fines del siglo XVII la región estaba poblada, por lo cual, debido a la actividad una última etapa desde 1976 hasta la actualidad. El proyecto fundacional de La Plata, como ciudad planificada puede resumirse en tres elementos básicos: el casco urbano, un cuadrado perfecto de unos 5,2 km por lado (Garnier, 1994) totalizando una superficie de aproximados 27 $\mathrm{km}^{2}$, el puerto y la zona de quintas y chacras, que siguiendo la proyección de las avenidas del casco y pasando la avenida de circunvalación, se situaba como un sistema de anillos de abastecimiento regional y local.

Mientras en la periferia norte las tierras fueron entregadas por el gobierno de la provincia o adquiridas a bajo costo por familias vinculadas con el poder político, también se asentaron inmigrantes portugueses y japoneses que se dedicaron a la producción de hortalizas y flores. En la periferia sur los propietarios de la tierra, en conjunto con las primeras inmobiliarias, 
realizaron la subdivisión de quintas originales en manzanas urbanas para que allí se asentara la población obrera, los talleres y pequeños comercios. Esta situación trajo aparejado un tejido urbano más denso y de terrenos pequeños (Frediani, 2010).

A partir de la década de 1930 debido a la masiva oleada migratoria que se produjo desde el interior del país hacia zonas con mayor desarrollo industrial, como parte de la política de sustitución de importaciones y del desarrollo del transporte automotor, el crecimiento urbano de la ciudad de La Plata comenzó a extenderse por fuera del casco. La subdivisión del suelo rural en pequeñas parcelas permitió el acceso masivo a la propiedad individual en áreas periféricas.

En la segunda etapa del modelo sustitutivo de importaciones, el crecimiento residencial comenzó a extenderse en condiciones precarias, carente de infraestructuras básicas y sobre áreas inundables. Es así como emerge una nueva forma de ocupación masiva de la tierra urbana: la ocupación ilegal de la tierra de migrantes procedentes de distintas partes de Argentina y países limítrofes sin ningún plan de urbanización ni de servicios públicos, implicando graves problemas para las personas y el ambiente (Frediani, 2010).

Desde la planificación territorial, en el año 1961 es elaborado el plan URBIS (Plan Regulador Conjunto para los Partidos de La Plata y Ensenada) que comprendía a dos municipios de la región y, entre sus objetivos, contaba con el desarrollo coordinado del área urbana de expansión de los tres partidos. Esta proponía la densificación del casco fundacional y la contención del crecimiento en las periferias, aunque en la práctica sólo sucedió lo primero. Entre 1976 y 1991, con el modelo aperturista importador, la actividad industrial se vio notoriamente diezmada en la región, especialmente en La Plata y Berisso. La desocupación y la precarización de los salarios condujeron a un aumento de la pobreza en la zona urbana, incrementándose los asentamientos precarios, surgiendo también otros hábitats informales sobre áreas no aptas para la urbanización.

En este período se promulga el decreto Ley de Ordenamiento Territorial y Usos del Suelo de la Provincia de Buenos Aires (1977) y un año después la Ordenanza Municipal n4495 de Zonificación según Usos y Normas de Subdivisión de Tierras en el Partido de La Plata, que tiene como finalidad adecuar la citada ley. También se produce un aumento poblacional en áreas periféricas y extraurbanas, desbordándose los límites propuestos en la Ordenanza $\mathrm{N}^{\circ} 4495$, y un leve decrecimiento en el Casco Fundacional (Frediani, 2010).

Mientras en el año 1990 el área urbana del Partido de La Plata ocupaba 4831 ha (Atlas CIPUV), en el año 2000 abarcaba 7815 ha, y en 2010 llegaba a 11172 ha, lo que significa un incremento porcentual similar en los dos períodos intercensales (38\% y 30\%, respectivamente). Sin embargo, el mayor incremento en el período 2000-2010 se asocia con la extensión en superficie de la zona urbana con un patrón fragmentado, lo que configura una zona que denominaremos periurbana, mientras que en el período 1990-2001 se incrementó alrededor de un $10 \%$ en el período 2000-2010 lo ha hecho casi un 52\%.

En relación con el área rural, el mismo estudio revela un incremento del $13 \%$ en el período $1990-2000$ y de un $25 \%$ en el siguiente período, acumulando un $35 \%$, porcentaje notoriamente inferior al de los incrementos de las superficies urbana y periurbana.

En la Figura 2 se visualiza el proceso de expansión urbana del GLP, desde el siglo XIX hasta la actualidad.

Por lo expuesto, ningún instrumento de planificación como un plan Director o un Plan Urbano Territorial planteó orientar el crecimiento urbano hacia lugares ambientalmente seguros. Esta interpretación surge de la escasa restricción a la ocupación en zonas inundables como las planicies de inundación, como así también de la falta de gestión y seguimiento de la ocupación de la zona rural por viviendas y por el aumento de invernaderos. La superficie cubierta por estos últimos se incrementó en forma exponencial en las últimas décadas alcanzando las 8000 ha cubiertas en 2016 (Diario El Día, 2016).

\section{Lo urbano, lo periurbano y lo rural en los últimos treinta años}

Lo urbano refiere a una ocupación y subdivisión 


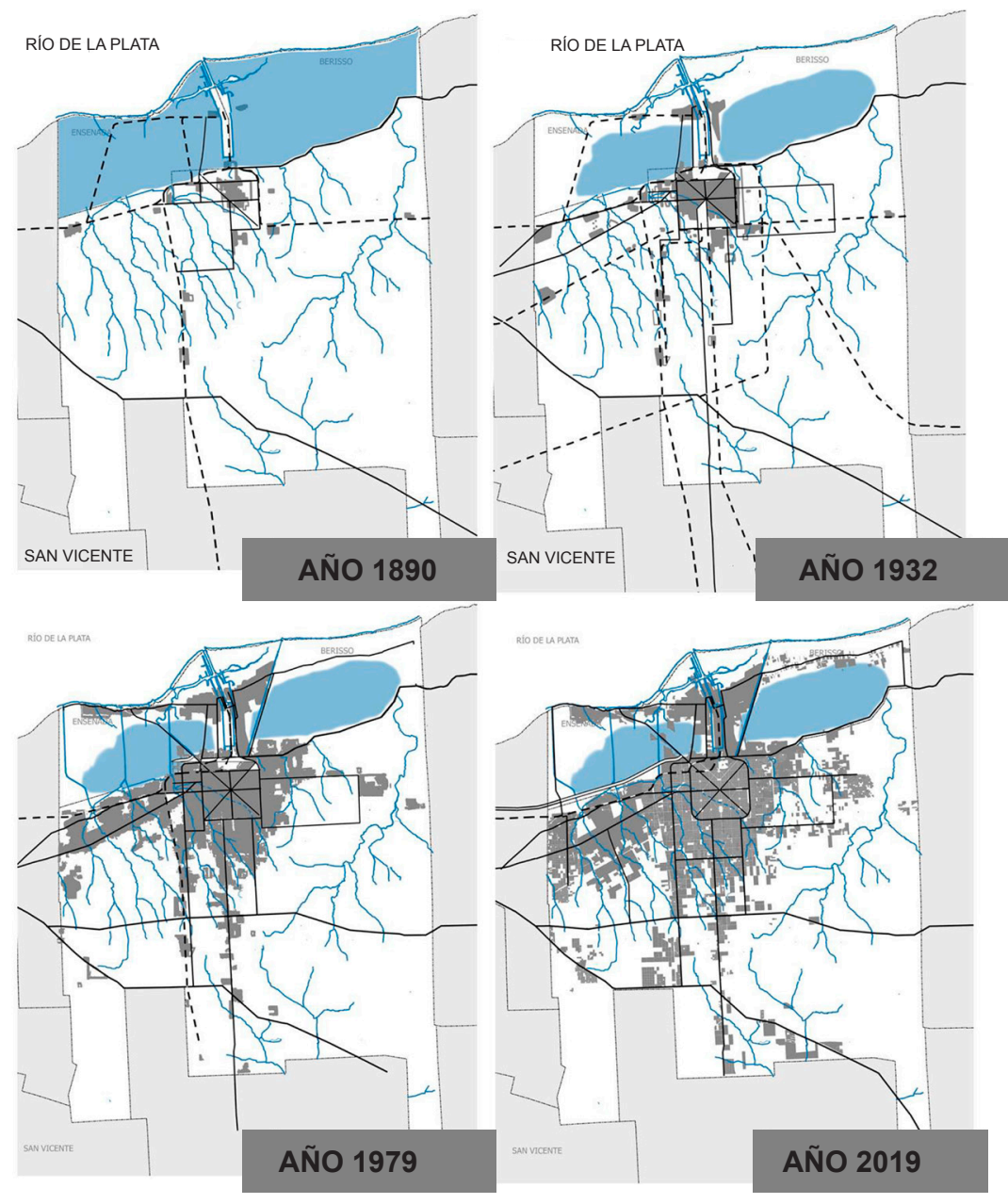

Figura 2. Expansión del área urbana del Gran La Plata. Fuentes: Elaboración de López, y Rodríguez Daneri (en base a López, 1992; Hurtado, M. A., Giménez, J. E., et al. (2006) y Plano de ocupación del Gran La Plata del Plan Director de Reducción de Riesgo de Inundaciones. La Plata; Plano de Ocupación del Gran La Plata (2019) Eugenia Rodríguez Daneri) Figure 2. Expansion of the urban area in Greater La Plata.

del suelo intensiva que aloja la casi totalidad de las actividades relacionadas con la producción de servicios; las de consumo y máximo intercambio de mercaderías o bienes, personas, finanzas e información. También la gestión y la administración pública, empresarial y comunitaria y los espacios abiertos públicos para el ocio y la recreación.

Lo rural se caracteriza por la presencia de grandes espacios abiertos dedicados al cultivo, la cría de animales y las instalaciones de apoyo a las actividades agrícolas.

Lo periurbano constituye la zona de transición entre lo urbano y lo rural. Racine (1967) acuñó el término "peri" en 1967 para designar periferias norteamericanas inconexas, y al proceso de formación de estas lo llamó "metamorfismo periurbano" cuyo rasgo sobresaliente es la desnaturalización de las estructuras urbanas (desurbanización) y rurales (desruralización). Sin embargo, hoy los espacios periurbanos parecen remitirse a la complejidad y escala espacial de los fenómenos urbanos donde estén ubicados (estructuras urbanas complejas, conurbaciones y/ o regiones urbanas). También a la valoración del espacio rural y al ambiente económico regional, sea este expansivo o recesivo, a las coyunturas locales y a las características de los grupos sociales dominantes.

Valenzuela Rubio (1985) define a los sectores periurbanos como: “...aquella pieza territorial que, aunque marcada por la presencia de lo 
urbano en múltiples versiones, aún se halla insertada en una matriz agraria pujante, funcional y paisajística. Su principal característica y condición es la de espacio de conflicto". Estos espacios están en constante pérdida de suelo agrícola, muchas veces de gran calidad agronómica, con una transformación difusa de medio rural a medio urbano y actividades urbanas. Como consecuencia de esto el suelo va perdiendo su condición de productor de rentas para convertirse en fondo de valor.

Es posible describir lo periurbano, como los espacios del entorno rural con implantación de elementos no agrícolas.

Otro concepto empleado en el país es el de "cinturón verde" que se refiere al periurbano formado por huertas o quintas familiares, algunas con características empresariales que rodean a las grandes ciudades, con una producción destinada a verduras de hoja y hortalizas de estación. Estas áreas han sido la primera manifestación de la horticultura en Argentina a fines del siglo XIX (Barsky, 2007). En el caso de La Plata el cinturón verde abastece 14 millones de habitantes dentro de la RMBA y también provee a otras zonas del país. En los últimos treinta años acaecieron grandes transformaciones tecnológicas en la actividad hortícola. Tres pilares explican estas transformaciones: los horticultores (principalmente de nacionalidad boliviana), los invernaderos y la explotación de la fuerza de trabajo (García, 2012).

Durante el período 1989-2001 se incrementó de países limítrofes y en particular procedente de Bolivia junto a la incorporación tecnológica del invernáculo, intentando buscar diferenciación del producto hortícola. La evolución hace que la superficie bajo esta forma desde su inicio en 1985 hasta el relevamiento llevado a cabo por el Censo Hortícola de Buenos Aires 1998 (CHBA'98), pero a partir de allí la tasa de crecimiento de la superficie bajo cubierta se ha incrementado exponencialmente a casi 61 has/ año (CHFBA' 05) (García, 2011)

Durante las últimas tres décadas en la RMBA se produjo la expansión de barrios de ingresos medios y altos, en algunos casos bajo la forma de barrios cerrados, acentuando los contrastes sociales de las áreas periféricas, y específicamente del periurbano, consecuencia de la incorporación de nuevas tierras a la ciudad (Barsky, 2005).

Como fenómeno reciente, situado aproximadamente en los diez últimos años, comienzan a generalizarse nuevas formas de habitar el periurbano, asociadas a sectores medios y bajos mediante el acceso a créditos blandos, específicamente el caso del Programa Crédito Argentino del Bicentenario (Pro.Cre.Ar). En la ciudad de La Plata esto se asocia con una rezonificación de tierra rural en tierra urbana para los beneficiarios de créditos Pro.Cre.Ar (Ordenanza N¹1094/13).

Indudablemente emergen del espacio periurbano múltiples conflictos y tensiones sociales que devienen de las interferencias entre distintas actividades que ocupan la estructura territorial, a partir de la pervivencia de usos agrarios compartiendo y compitiendo por el espacio con diversos usos urbanos, muchas veces degradantes del medio.

El periurbano es sin dudas un espacio de oportunidad para poner en práctica conocimientos que brinda la ecología, como considerarla un área de amortiguación entre lo urbano y lo rural. En este caso específico las nacientes de arroyos perteneciente a la vertiente del estuario del Río de la Plata, se sitúan en su mayoría en este espacio, sumándole mayor complejidad, por la contraposición que implica el avance del uso residencial y lo perjudicial que puede resultar la producción hortícola bajo cubierta por la impermeabilización que produce del suelo, sobre tramos bien conservados de arroyos y humedales.

\section{Cuencas, humedales y arroyos en el Gran La Plata}

Los arroyos del Gran La Plata, nacen en el partido homónimo, en bañados y/o cañadas ubicadas en la divisoria de aguas del río Samborombón y forman parte de la cuenca sur del Río de la Plata. Coinciden, en parte, con la zona rural del mismo partido, actualmente permanecen ocultos $y / 0$ transformados y atraviesan zonas rurales, periurbanas y urbanas. En este último tramo muchos están entubados, originando problemas de drenaje, anegamientos e inundaciones que el 2 de abril de 2013 tuvieron su máximo pico. Todos estos cursos llegan al Río de la Plata 
mediados por una zona muy baja denominada planicie de inundación, que se ubica entre el litoral urbanizado de Berisso y Ensenada y la zona más alta de la pampa ondulada donde se despliega tanto la urbanización compacta como la dispersa y la zona rural de La Plata, atravesada por diez subcuencas y sus respectivos arroyos, que tienen origen en la zona alta (30 y $25 \mathrm{~m}$ s.n.m.) de La Plata (Figura 3).

Por mucho tiempo ríos y arroyos fueron considerados obstáculos para la conectividad y/o sumidero para el vuelco de desechos de algún proceso manufacturero o industrial.

Algunas zonas más altas y seguras entre subcuencas o divisorias de aguas fueron utilizadas para fundar pequeños pueblos, como lo solicitaba la ley de fundación de pueblos, que armó una estructura regional de base. Se sumaron hasta 1885, Ensenada, Berisso, Tolosa, La Plata y el puerto y hasta 1927 , sobre el trazado de las vías del ferrocarril, Villa Elisa, Abasto, City Bell, Melchor Romero; Olmos, Gorina y Seguí, y solo estaciones de ferrocarril en Gonnet y Ringuelet. Entre ellos discurrían diferentes arroyos que atraviesan el partido. Asentamientos como Los Hornos, Villa Elvira; Villa Arguello, Altos de San Lorenzo, San Carlos se suman a la estructura territorial a partir de subdivisiones del suelo cercanos a grandes equipamientos y/o a fuentes de trabajo. Luego se inicia la urbanización por extensión de subdivisiones de suelo de tipo urbano que ampliaron los trazados originarios de los pueblos mencionados. Llegando a parcelarse y ocupar las planicies de inundación de los arroyos. Entre 1960 y 1976 con el levantamiento de algunos ramales del ferrocarril se desarrolla más intensamente el transporte automotor público y privado. Esto permitió llegar a los puntos más alejados del territorio. En estos procesos los arroyos que separaban las urbanizaciones fueron conformando bordes/ barreras y cuando estas se extendieron, los arroyos utilizados como drenajes, se entubaron, y, algunos de los espacios de evacuación de efluentes causaron inundaciones porque no fueron tratados con la lógica de ocupación de los márgenes o riberas, ni respetando su cauce ni adaptándose a su cuenca; y lo que es peor, se subdividió y ocupó la planicie de inundación o los espacios de ampliación de sus cauces. Hoy cada uno de ellos divaga en la subcuenca de pertenencia conformando un sistema de drenaje, en algunos tramos entubados o funcionando a cielo abierto, algunos muy contaminados y drenando por canalizaciones al Río de la Plata.

\section{Planificación reciente del territorio}

En las últimas tres décadas se permitió subdividir el suelo en parcelas urbanas para uso residencial sin dotación de infraestructura básica a modo de grandes superficies o en parcelas rurales con subdivisiones en propiedad horizontal; sin tener en cuenta el paso de los arroyos, muchas veces incluidos al interior de las superficies cuando ellos son de carácter público (clubes de campo; barrios cerrados u otros modelos de urbanización semejantes) que extendieron la ocupación residencial por expansión a lo largo y ancho del Partido incluso en zonas rurales. Así se abrió un nuevo frente de urbanización sobre la Ruta 2 como zona exclusiva para clubes de campos. Como contracara, al mismo tiempo aumentaron exponencialmente los asentamientos informales. Los primeros privatizando los arroyos como parte del paisaje, así como los humedales y cañadas de las nacientes y los segundos ocupando sus orillas y planicies de inundación.

El concepto de humedal puede definirse como biotopos en tierras bajas, cubiertas temporaria o intermitentemente por aguas poco profundas; la combinación de condiciones acuáticas y terrestres los producen e identifican como humedales (Bacon, 1996). Esto hace de ellos ecosistemas complejos determinados por procesos hidrológicos, que pueden tener fluctuaciones diarias, estacionales, o a largo plazo, relacionadas con el clima regional y la ubicación geográfica.

En el Gran La Plata surge el primer tratamiento especial de una zona litoral pensada como ecosistema en 1940 denominada Reserva Natural de Punta Lara, aunque no se la reconoce como humedal. Estas tierras se adicionaron en el año 2002 las tierras correspondientes al Parque Pereyra por la Ley $N^{\circ} 12814$ conformando una Reserva Natural Integral Mixta. Por otra parte 


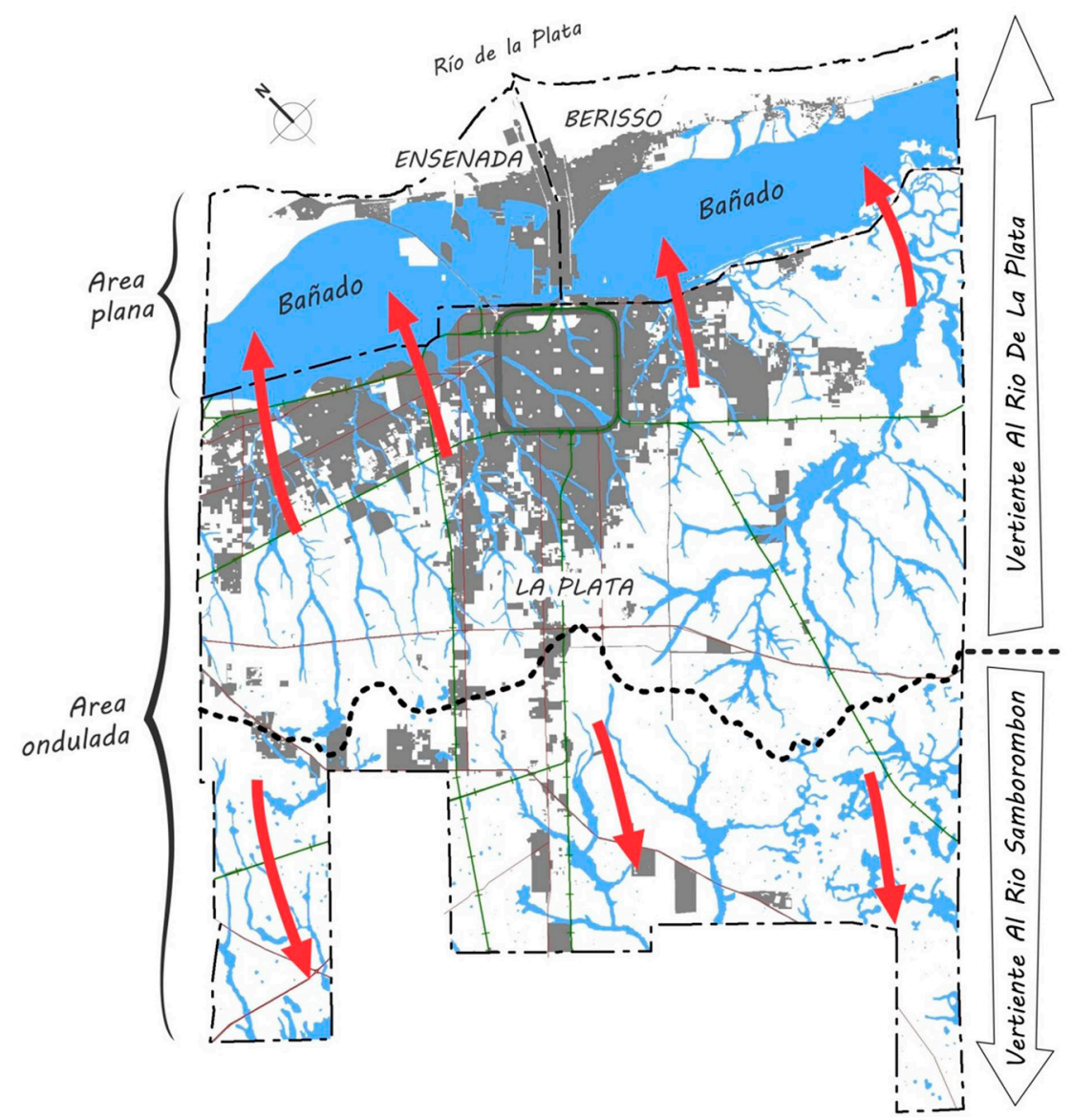

Figura 3. Cuencas del Gran La Plata. Fuente: IGS-CISAUA, 2017.

Figure 3. Basins of the Greater La Plata.

la cuenca del Arroyo el Pescado fue declarada Paisaje Protegido de Interés Provincial en 1998 aunque su cuenca aún no ha sido delimitada efectivamente. Otro humedal que ha sido protegido en 2001 es el Monte Ribereño de la Isla Santiago, declarado como Paisaje Protegido de Interés Provincial. Todos ellos reconocen indirectamente ser humedales de tipo costero. Sin embargo como ya se enunció también existen los interiores.

Desde las normativas municipales de uso del suelo por Ordenanza No 9231/2000 La Plata reconoce por primera vez como zonas especiales los arroyos cuando atraviesan el periurbano disponiendo su protección donde aún están a cielo abierto aunque en zonas urbanas siguen entubados. En 2010 se modifica esta ordenanza de usos del suelo por Ordenanza N 10701/10, que continúa previendo restricciones para edificar en el margen de las zonas de arroyos, pero sin reconocer ni la planicie de inundación, ni el periurbano como una zona especial. Tampoco hace obligatorio la extensión de servicios esenciales para evitar la degradación ambiental, ni reconoce la necesidad de tratamiento de los asentamientos informales que aumentaban en cantidad y extensión en los márgenes de arroyos del Gato y Maldonado.

Tanto en Berisso como en Ensenada poseedores de un gran humedal en su territorio, aún no han sido reconocidos como tales. En 1978 el bañado de Maldonado en Ensenada es declarado zona rural (Ordenanza N 866/78) y a su vez ese mismo año se expropia parte de él para emplearlo con rellenos sanitarios, para lo cual se creó la empresa estatal Coordinación Ecológica Área Metropolitana Sociedad del Estado (CEAMSE), haciendo un uso indebido del humedal, que continua. En Berisso, las 
normativas de uso del suelo reconocen el mismo bañado como área a recuperar para usos urbanos.

Todo esto demuestra que, si bien históricamente se fueron reconociendo algunos humedales, solo son los que limitan con el litoral y como espacio de naturaleza, pero aún no se consideran como espacios de convivencia entre sistema agrario -humedal o sistema urbano - humedal. La preservación de recursos naturales para la sustentabilidad de territorios y ciudades y el desarrollo de sistemas alimentarios de proximidad como los del periurbano, otorga una oportunidad, pero aún no están suficientemente desarrollados, aunque tanto INTA, organizaciones AUPA y Pro Huerta, aun lo consideran como políticas en este sentido.

Para esto surgen las políticas de adaptación al medio natural que puede reconocer según las estrategias que sean necesarias. Entre ellas sobresalen la conservación y/o preservación, cuando aún los humedales no hayan sido modificados; la restauración cuando hayan sido transformados y puedan seguir prestando servicios de infiltración y/o de nacimiento de los arroyos como en el caso de La Plata. Esto permitiría hacer notar y definir donde y como tienen su origen para incorporarlos como un acontecimiento territorial entre lo rural y lo urbano, e incorporarlo como política integral del agua y mostrar a la población su importancia en el marco de las estrategias que enfrenten las posibles inundaciones. Por último, la creación o construcción, cuando se trata de humedales intervenidos con grado diferencial de deterioro, desde la alteración de sus funciones fundamentales, hasta su extinción y finalmente su evaluación, aunque hay diferentes opiniones con referencia a la ventaja de estas acciones.

\section{Estrategias para la protección de los bañados en la región}

Muchas veces el ámbito territorial adecuado para un tratamiento integrado de los espacios periurbanos desborda la jurisdicción municipal, especialmente en las grandes ciudades y en las regiones metropolitanas. Por ello, para su ordenamiento y gestión, se requieren diseñar figuras o normas urbanísticas complejas (directrices territoriales, por ejemplo) en los ámbitos sub o supramunicipal según los casos, en donde confluyan la escala adecuada de los problemas con los instrumentos capaces de afrontarlos.

Su tratamiento por lo tanto es particularmente difícil, hasta el punto de que para algunos constituye el gran reto del urbanismo moderno. $Y$ en ese caso merece la pena detenerse algo más en el tema de la implantación de los usos recreativos, pues todo lo que suponga reforzar el mejoramiento ambiental, será favorable para mantener su condición como espacios de privilegio para alojarlos.

Lo que se impone por sobre todas las cosas, es el tratamiento integrado de los espacios rurales, periurbanos y urbanos. A partir de la escala de los problemas territoriales que sostienen, la necesidad de crear marcos legales y administrativos adecuados a su especificidad resulta imprescindible. A ello no podrá llegarse, sin una reflexión científica capaz de identificar las raíces profundas de los problemas. Y además debe hacerse explícita la voluntad de intervenir en la reconducción de los conflictos, situación que tendrá que ir acompañada de programas informativos y respuestas alternativas.

En 2017 y a partir del desarrollo de un proyecto de investigación que intento conocer y dar respuesta a la gran inundación de 2013, se propusieron lineamientos para la mejora ambiental (Figura 4) creando previamente tantas unidades de gestión como subcuencas haya para luego cumplir con las medidas que dispongan las diferentes estrategias tanto para la conservación, adaptación y recuperación de humedales, a saber:

- Conservar los cauces de los arroyos aún abiertos como corredores ecológicos públicos, procurando trazar los caminos de sirga (aún en casos que no figuren en el catastro territorial) para que cumplan un rol múltiple: respectar los márgenes de los arroyos previendo la inundación; trabajar con la población en la percepción y respeto de estos espacios, como lugares de infiltración, sendas, paseos y corredores urbanos de nuevo recorrido que amplían y mejoran la conectividad 
actual (en áreas urbanas, periurbanas y rurales) - Conservar los humedales que aun permanezcan en estado natural y dan origen a los arroyos creando un sistema de áreas protegidas municipales y recuperar los humedales actualmente degradados pero no ocupados, incorporándolos como suelo natural y contenidos por un área buffer que puede ser incorporado a la práctica de cultivos especiales. Pueden incorporarse zonas atravesadas por cauces de arroyos que aun sean naturales.

- Conservar los bañados correspondientes a Ensenada y Berisso como parque inundable natural y apoyo a la educación ambiental. Modificando la normativa municipal y creando circuitos de paseos para que sean conocidos y defendidos por la misma población. - Promover la conservación de suelo rural en La Plata a partir de una estrategia de creación de un parque agrario en el cinturón fruti-flori-hortícola como espacio de desarrollo económico - productivo que también protege y/o recupera todos los bañados que dan origen a los arroyos.

- Crear con todos los bañados Protegidos y/o Recuperados un itinerario que será parte del Parque Agrario como sistema de Parques Municipales Educativos Húmedos. Entendiéndolo no como un parque al estilo tradicional delimitando un área, sino como con estrategias que se entienden como varios circuitos interrelacionados a través de uno o dos nodos, que incentiven la posibilidad de asociar trabajo agrario, recreación y educación ambiental $y$, que tiene como principal eje relacionar múltiples actividades, incluidas las huertas familiares, el vino de la costa de Berisso y/o la variedad de Paisajes Protegidos Costeros. - $\quad$ Adaptar y controlar la ocupación del suelo con invernaderos para la recuperación del agua de lluvia del predio que ocupan y preservar el acuífero Puelche.

- Recuperar como suelo rural las zonas atravesadas por cauces de arroyos actualmente desocupadas que, por normativa municipal, están reservadas para urbanizar.

- Retardar el flujo de las aguas pluviales creando parques inundables en espacios estratégicos de los cauces de los arroyos y sus planicies de inundación. Hasta el momento desde la Dirección de Hidráulica de la Provincia de Buenos Aires se está trabajando en la creación de seis parques, la mayoría en humedales linderos a los arroyos.

- $\quad$ Adaptar las construcciones delimitadas en la zonificación de riesgo hídrico con la participación de la comunidad afectada (reviendo Código de Edificación).

- Adaptar el Factor de Ocupación del Suelo (FOS) actual, limitando con indicadores importantes la ocupación de cada parcela.

- $\quad$ Adaptar los parques y plazas existentes como parques inundables.

- Rediseñar veredas y pavimentos con materiales que permitan la infiltración del agua pluvial.

- $\quad$ Promover la arborización equilibrada con especies nativas en los márgenes de los arroyos en áreas rurales y/o periurbanas.

Para cumplir con esta gran tarea, además de necesitar un marco jurídico y presupuestario, deberían tener un entrenamiento adecuado los funcionarios responsables de aspectos ambientales, a niveles local y regional, así como el establecimiento de políticas de manejo ambiental en función de las áreas naturales de influencia de los humedales y no de los límites arbitrarios determinados por consideraciones políticas o sectoriales.

Por último, y no por eso menos importante, la conservación de la biodiversidad debe compatibilizarse con las actividades de desarrollo. En este caso las actividades rurales intensivas y la urbanización con desarrollo equitativo y equilibrado. Planes de desarrollo que consideren esferas de influencia espacial, temporal y social, que excedan su localización, y el uso actual del área afectada, requiriendo, además, la participación de amplios sectores de la comunidad.

Muchas veces con intervenciones espacialmente puntuales; pero con efectos que puedan trascender ampliamente su localización y compatibilizarse con una gestión integrada de los recursos hídricos, basada en la consideración de qué cada subcuenca hidrográfica necesita un abordaje ambiental especial para ella. 


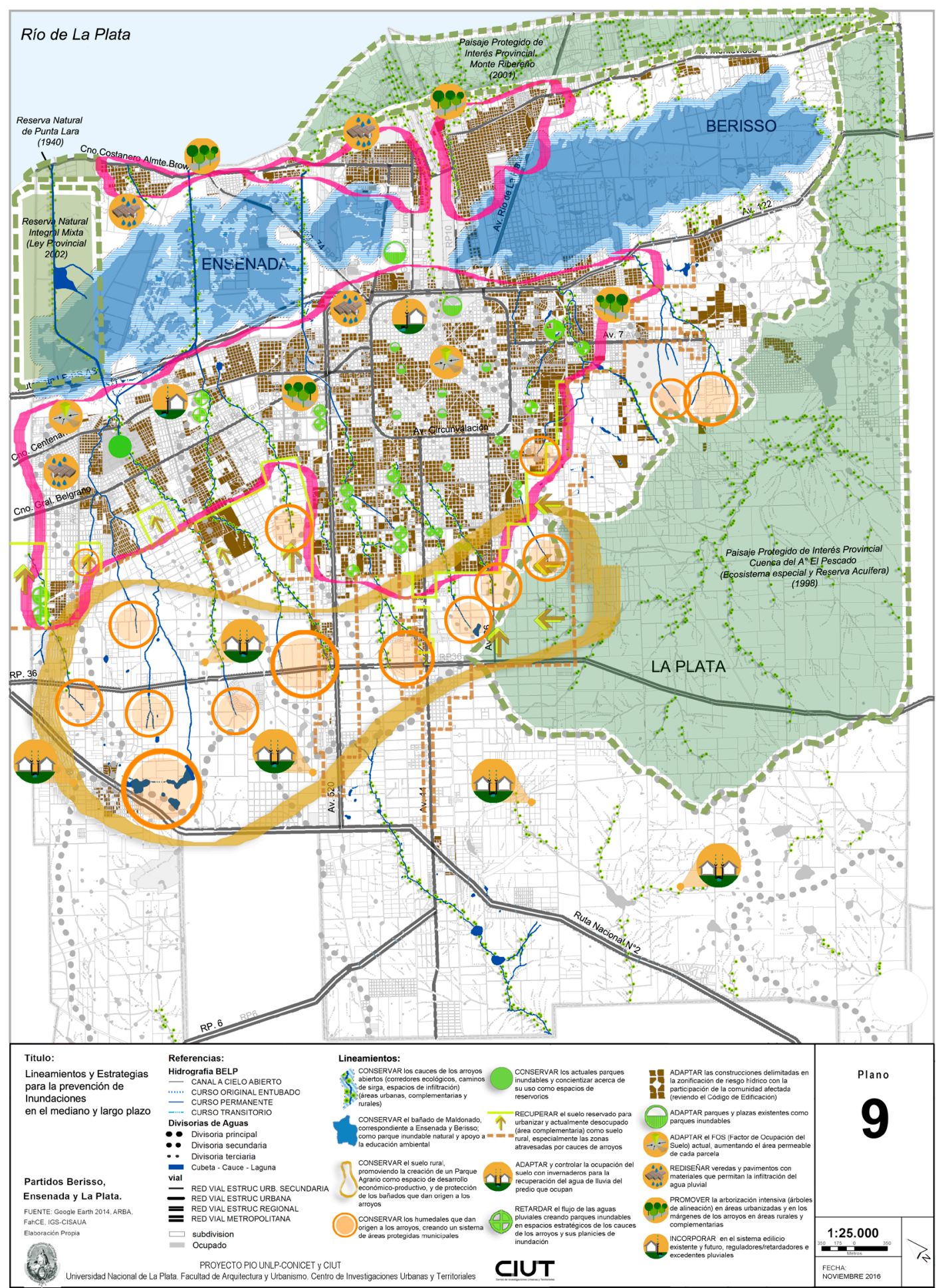

Figura 4. Lineamientos y Estrategias para la adaptación a las inundaciones. Fuente: Ronco y López, 2017

Figure 4. Guidelines and strategies for adaptation to floods. 


\section{REFERENCIAS}

Atlas CIPUV de Precios del Suelo de la Región Metropolitana de Buenos Aires. Recuperado en: http://atlas-cipuv.utdt.edu/\#/ (fecha de acceso: 30-01-2020).

Bacon, P. (1996). Wetlands and biodiversity. In: Hails, A. J. (Ed.). Wetlands, Biodiversity and the Ramsar Convention: The Role of the Convention of Wetlands in the Conservation and Wise Use of Biodiversity. Gland: Ramsar Convention Bureau.

Barsky, A. (2005). El periurbano productivo, un espacio en constante transformación. Introducción al estado del debate, con referencias al caso de Buenos Aires. Scripta Nova, 9(194), 36.

Barsky, A. (2007). La agricultura de "cercanías" a la ciudad y los ciclos del territorio periurbano. Reflexiones sobre el caso de la Región Metropolitana de Buenos Aires. En: Svetlitza de Nemirovsky, A. (Coord.). Globalización y agricultura periurbana en la Argentina. Escenarios, recorridos y problemas. Maestría en Estudios Sociales Agrarios. Serie Monografías. (pp 15-29). Buenos Aires: FLACSO.

Diario El Día. (2016). Alerta por el crecimiento descontrolado de invernaderos en el Gran La Plata Recuperado en: https://www.eldia.com/ nota/2016-11-13-alerta-por-el-crecimientodescontrolado-de-invernaderos-en-el-gran-laplata (fecha de acceso: 30-01-20)

Frediani, J. (2010). Lógicas y tendencias de la expansión residencial en áreas periurbanas. El Partido de La Plata, Buenos Aires, Argentina, entre 1990 y 2010 (Tesis doctoral, Facultad de Humanidades y Ciencias de la Educación, UNLP).

García, M. (2011). El cinturón hortícola platense: ahogándonos en un mar de pláticos: Un ensayo acerca de la tecnología, el ambiente y la política. Theomai: estudios sobre sociedad, naturaleza y desarrollo, 23, 3-19.
García, M. (2012). Análisis de las transformaciones de la estructura hortícola platense en los últimos 20 años. El rol de los horticultores bolivianos. (Tesis doctoral. Faculta de Ciencias Agrarias y Forestales. UNLP).

Garnier, A. (1994). El cuadrado roto: sueños y realidades de La Plata. La Plata: Municipalidad de La Plata.

Hurtado, M. A., Giménez, J. E., et al. (2006). Análisis ambiental del partido de La Plata. Aportes al ordenamiento territorial. Buenos Aires, Argentina: Consejo Federal de InversionesMunicipalidad de la Plata.

IGS-CISAUA (2017). Las inundaciones en la Región Capital - Cartografía Temática para el Planeamiento. En: Las inundaciones en La Plata, Berisso y Ensenada: análisis de riesgo, estrategias de intervención. Hacia la construcción de un observatorio ambiental. (pp. 2-34). (Disponible en: http://sedici.unlp.edu.ar/ handle/10915/59633. (fecha de acceso: 3001-2020)

INDEC - INSTITUTO NACIONAL DE ESTADÍSTICA Y CENSOS (2010). Censo nacional de Población y Vivienda. Disponible en: https://www.indec. gov.ar/nivel4_default.asp?id_tema_1=2\&id_ tema_2=41\&id_tema_3=135. (Fecha de acceso en: 10-03-2020).

López, I. (1992). Emergentes de los Planes urbanos y nuevas Estrategias. Estudio de Caso Partido de La Plata. En A.A.V. V.: La Plata: de la ciudad antigua a la ciudad nueva. Sueños y realidades, La Plata: Municipalidad de La Plata.

López, I., Etulain, J. C. et al. (2016). Inundaciones urbanas: mapas de riesgo y lineamientos de ordenamiento urbano territorial. En: Ronco, A. y López, I. Las inundaciones en La Plata, Berisso y Ensenada: análisis de riesgo, estrategias de intervención. Hacia la construcción de un observatorio ambiental. (pp.1-37). Recuperado en: http://sedici.unlp. edu.ar/handle/10915/59633 (fecha de acceso: 29-04-2020) 
Racine, J. B. (1967). Exurbanisation et métamorphismepériurbain: introduction à l'étude de la croissance du Grand Montréalau sud du Saint-Lauren". Revue de géographie de Montréal, 21(2), 313-342.

Ronco, A., y López, I. (2017). Las inundaciones en La Plata, Berisso y Ensenada: análisis de riesgo, estrategias de intervención. Hacia la construcción de un observatorio ambiental. Cartilla de Divulgación. Recuperado en: http://sedici. unlp.edu.ar/bitstream/handle/10915/59633/ Cartilla_de_divulgaci\%C3\%B3n.pdf-PDFA. pdf? sequence=64\&isAllowed=y. (fecha de acceso en: 10-03-2020).
Rotger, D. V. y López, I. (2019). El paisaje detrás de las diagonales. Vínculos entre arroyos y la urbanización en la ciudad de La Plata. Estudios del hábitat, 17(1), 2-16.

Rotger, D., Aversa, M. y Jáuregui, E. (2018). Cambio climático, inundaciones y "lagunas" de información. Análisis de inundaciones a través del rastreo de artículos periodísticos en el Gran La Plata (Buenos Aires, Argentina). Cadernos Metrópole, 20(42), 305-324.

Valenzuela Rubio, M. (1985). Los espacios periurbanos. En: IX Coloquio de Geógrafos Españoles. Actas, discursos, ponencias, y mesas redondas. (pp. 81-123). Murcia: Relatoría de las Comunicaciones. 